\title{
Polarimetry as a tool to find and characterise habitable planets orbiting white dwarfs
}

\author{
Luca Fossati ${ }^{1} \dagger$, Stefano Bagnulo ${ }^{2}$, Carole A. Haswell ${ }^{3}$, \\ Manish R. Patel ${ }^{3}$, Richard Busuttil ${ }^{3}$, Piotr M. Kowalski ${ }^{4}$, \\ Denis V. Shukyak ${ }^{5}$, Michael F. Sterzik ${ }^{6}$ and Gennady Valyavin ${ }^{7}$ \\ ${ }^{1}$ Argelander-Institut für Astronomie der Universität Bonn, \\ Auf dem Hügel 71, 53121 Bonn, Germany \\ email: Ifossati@astro. uni-bonn.de \\ ${ }^{2}$ Armagh Observatory, College Hill, Armagh BT61 9DG, Northern Ireland, UK \\ ${ }^{3}$ Department of Physical Sciences, The Open University, Walton Hall, \\ Milton Keynes MK7 6AA, UK \\ ${ }^{4}$ Institute of Energy and Climate Research (IEK-6), Forschungszentrum Jülich, \\ Wilhelm-Johnen-Strasse, D-52425 Jülich, Germany \\ ${ }^{5}$ Institute of Astrophysics, Georg-August-University, Friedrich-Hund-Platz 1, \\ D-37077, Göttingen, Germany \\ ${ }^{6}$ European Southern Observatory, Karl-Schwarzschild-Strasse 2, D-85748 Garching, Germany \\ ${ }^{7}$ Special Astrophysical Observatory of the RAS, 369167, Nizhny Arkhyz, \\ Karachaevo-Cherkesia, Russia
}

\begin{abstract}
There are several ways planets can survive the giant phase of the host star, hence one can consider the case of Earth-like planets orbiting white dwarfs. As a white dwarf cools from $6000 \mathrm{~K}$ to $4000 \mathrm{~K}$, a planet orbiting at $0.01 \mathrm{AU}$ from the star would remain in the continuous habitable zone $(\mathrm{CHZ})$ for about $8 \mathrm{Gyr}$. Polarisation due to a terrestrial planet in the $\mathrm{CHZ}$ of a cool white dwarf (CWD) is $10^{2}\left(10^{4}\right)$ times larger than it would be in the habitable zone of a typical M-dwarf (Sun-like star). Polarimetry is thus a powerful tool to detect close-in planets around white dwarfs. Multi-band polarimetry would also allow one to reveal the presence of a planet atmosphere, even providing a first characterisation. With current facilities a superEarth-sized atmosphereless planet is detectable with polarimetry around the brightest known CWD. Planned future facilities render smaller planets detectable, in particular by increasing the instrumental sensitivity in the blue. Preliminary habitability study show also that photosynthetic processes can be sustained on Earth-like planets orbiting CWDs and that the DNA-weighted UV radiation dose for an Earth-like planet in the CHZ is less than the maxima encountered on Earth, hence white dwarfs are compatible with the persistence of complex life from the perspective of UV irradiation.
\end{abstract}

Keywords. Polarisation, astrobiology, techniques: photometric, (stars:) white dwarfs

\section{Introduction}

The search for habitable Earth-like planets is a major contemporary goal of astronomy. The methods currently used for the detection of exoplanets is biased towards systems with small differences in mass, radius, and luminosity between star and planet (see e.g. Haswell 2010). For this reason M-type main sequence stars have become prime targets in the search of Earth-like planets in the habitable zone, which is about 0.1 AU away from the host star (see e.g. Selsis et al. 2007). M dwarfs evolve very slowly and their planets

$\dagger$ Also: Department of Physical Sciences, The Open University, Walton Hall, Milton Keynes MK7 6AA, UK. 


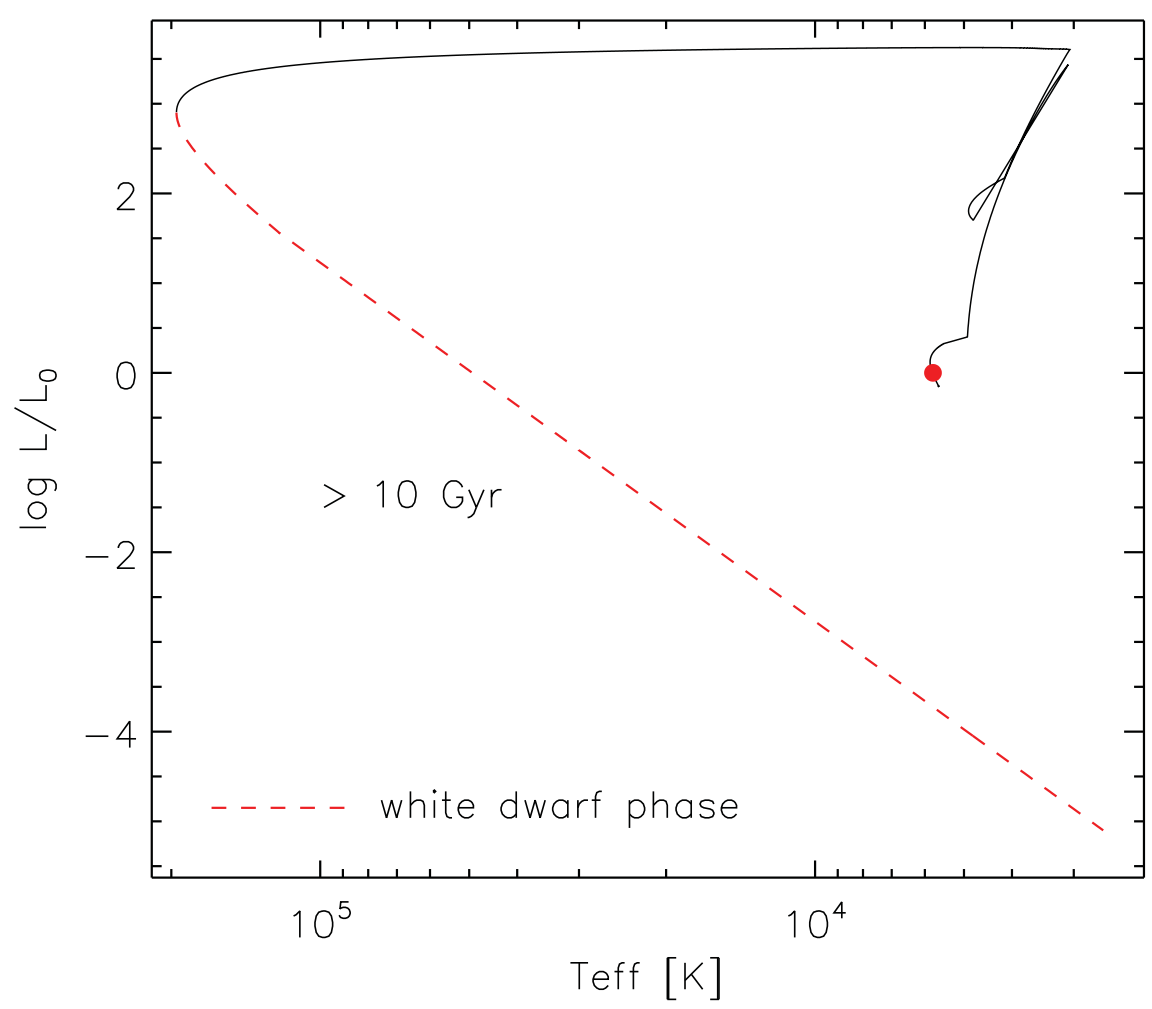

Figure 1. Evolutionary track in the HRD of a $1 M_{\odot}$ star calculated with the STARs stellar evolution code (Eggleton 1971; Stancliffe et al. 2007). The red dot indicates the current position of the Sun. The white dwarf phase, marked by a red dashed line, lasts for more than $10 \mathrm{Gyr}$.

might remain within a continuously habitable zone $(\mathrm{CHZ}) \dagger$, i.e. harboring surface liquid water, for several Gyr, providing ample time for the advent of life on a rocky planet.

With an effective temperature $\left(T_{\text {eff }}\right) \leqslant 6000 \mathrm{~K}$, cool white dwarfs (CWD) are also promising hosts of rocky planets in the habitable zone (Monteiro 2010; Agol 2011; Fossati et al. 2012; Barnes \& Heller 2013). These stellar remnants represent the final stage of the evolution of stars having an initial mass smaller than about $8 M_{\odot}$, namely almost all planet-hosting stars known to date. Figure 1 shows the evolutionary track of a $1 M_{\odot}$ star in the Hertzsprung-Russell diagram (HRD) calculated using the sTARs stellar evolution code (Eggleton 1971; Stancliffe et al. 2007). The white dwarf evolutionary phase is illustrated in Fig. 1 by the approximately straight red dashed line going from the top-left corner to the bottom-right corner of the HRD. The whole white dwarf phase lasts for more than 10 Gyr.

White dwarfs initially cool down rapidly, with the temperature decreasing by thousands of degrees in $\sim 3$ Gyr (see e.g. Salaris et al. 2010 ). At $T_{\text {eff }} \sim 6000 \mathrm{~K}$, crystallisation occurs, slowing down the cooling process. Agol (2011) showed that this leads to the formation of a habitable zone, which may last for up to $8 \mathrm{Gyr}$, well in excess of the time required for life to arise on Earth. In contrast to what occurs for M dwarfs, white dwarfs provide a stable luminosity source without the presence of potentially damaging radiation produced by stellar activity. In addition, because a planet orbiting close to a white dwarf would not

$\dagger$ Range of planet orbital distances at which the planet is habitable for a minimum of 3 Gyr. 
rise tides on the star (Agol 2011) it would synchronise within $1000 \mathrm{yr}$ and would have a stable orbit.

Recently, Valyavin et al. (2014) showed that convection is completely suppressed starting from surface magnetic fields of the order of $10 \mathrm{MG}$, and therefore strong magnetic fields slow down the white dwarf cooling process. As a consequence, the CHZ for strongly magnetic white dwarfs may extend even more in time, well beyond $8 \mathrm{Gyr}$. On the other hand, a planet orbiting a strongly magnetic white dwarf would permanently sit inside a magnetic field which may be as large as $1 \mathrm{kG}$. Depending upon the planet composition, this would lead to internal heating of the planet which, in some cases, might end up being too strong for the survival of the planet itself (see e.g. Li et al. 1998; Lai 2012). At the same time, it is not clear whether the presence of a permanent magnetic field as strong as $1 \mathrm{kG}$ would hamper the advent of life on an Earth-like planet. All these issues will be the subject of future works currently in preparation.

Due to the white dwarfs' small radius $\left(\sim 1 \mathrm{R}_{\oplus}\right)$, the low luminosity of CWDs creates a habitable zone at only $\sim 0.01 \mathrm{AU}$, ten times closer than for $\mathrm{M}$ dwarfs. This facilitates the detection of small bodies orbiting white dwarfs: Agol (2011) showed that transits of Mars-sized planets in the white dwarf $\mathrm{CHZ}$ would be $1 \%$ deep, hence easily detectable with present-day ground-based facilities, even for rather faint stars. Despite this, searches for planetary companions to white dwarfs have so far been unsuccessful (Friedrich et al. 2007; Faedi et al. 2011; Fulton et al. 2014).

\section{Habitability and possible presence of Earth-like planets orbiting cool white dwarfs}

Fossati et al. (2012) showed that the fluxes of a $5800 \mathrm{~K}$ white dwarf and of the Sun, integrated over the wavelengths playing a role in photosynthetic processes (see e.g. McCree 1972), are almost identical. This direct comparison is valid because the angular diameter of a CWD, as seen from the CHZ, is similar to that of the Sun, as seen from Earth (Agol 2011). In addition, the photosynthetic relevant flux intercepted by a planet in the $\mathrm{CHZ}$ of a $4000 \mathrm{~K}$ white dwarf is larger than that intercepted by the same planet in the habitable zone of a typical M dwarf (Raven 2007; Fossati et al. 2012). Photosynthetic processes would therefore be both feasible and efficient on planets orbiting in the white dwarf CHZ.

Using the code described in Patel et al. (2014), Fossati et al. (2012) showed also that the DNA-weighted UV dose encountered at the surface of an Earth-like planet in the white dwarf CHZ is comparable to that of an exoplanet in the habitable zone of a main sequence star at approximately $5000 \mathrm{~K}$. They finally concluded that the DNA-weighted dose for a hypothetical Earth-like planet around a CWD is remarkably benign from an astrobiological perspective, for an extremely long period of time.

A non-magnetic CWD is therefore a plausible source of energy for the advent and persistence of complex life, as we know it on Earth, but can rocky planets survive the whole main sequence and giant phase of the host star, and finally end up in a very close-in orbit around a white dwarf? Faedi et al. (2011) review several mechanisms which would result in a planet orbiting a white dwarf. Recently, Veras \& Gänsicke (2014) performed numerical simulations of the evolution of planetary systems, up to the white dwarf phase of the host star. They concluded that systems with closely-packed planets, with stable orbits during the main sequence phase of the host star, may experience inward planetary incursions during the white dwarf cooling phase. In this way, rocky planets might naturally end up in meta-stable close-in orbits around white dwarfs. Planets have also been found to orbit evolved stars: red giants (e.g. Frink et al. 2002; Sato et al. 
2003; Hatzes et al. 2005), sub-dwarf (e.g. Charpinet et al. 2011; Silvotti et al. 2014), and horizontal branch (e.g. Silvotti et al. 2007) stars. Mullally et al. (2008) reported also the detection of $\mathrm{a} \sim 2 \mathrm{M}_{J}$ planet in a $4.5 \mathrm{yr}$ orbit around a pulsating white dwarf.

Further evidence for the existence of rocky bodies close to white dwarfs comes from the presence of metallic lines (e.g. Si, Mg, and Fe) in the spectra of DZ white dwarfs (Zuckerman et al. 2003, 2010; Koester et al. 2014). Heavy metals in the atmosphere of these stars can only be explained by atmospheric "pollution" caused by the accretion of terrestrial-like material present in circumstellar disks (Gänsicke et al. 2006; Farihi et al. 2009, 2012; Melis et al. 2012; Veras \& Gänsicke 2014) or planetesimals (Bergfors et al. 2014). The circumstellar disks are thought to originate from the disruption of planets or planetesimals (e.g. asteroids) (Bonsor et al. 2011; Debes et al. 2012; Bear \& Soker 2013; Frewen \& Hansen 2014; Veras et al. 2014).

\section{Polarised light from an Earth-like planet in the white dwarf CHZ}

Agol (2011) made the case for transit searches targeting white dwarfs as in this way one could detect planets in the white dwarf CHZ. In the case of non-transiting planets, the "classical" planet finding methods would instead fail. Fossati et al. (2012) showed that the white dwarf CHZ is too close to the star to be detected by direct imaging. This is also the case of astrometric techniques: GAIA's astrometric precision could only allow one to detect an Earth-like planet in the $\mathrm{CHZ}$ for $0.6 M_{\odot}$ white dwarfs less than half the distance of Proxima Centauri. Microlensing too, is only sensitive to planets with wide orbits. The precision of radial velocity measurements would also not be enough to detect planets in the habitable zone of CWDs. This is because for hydrogen and helium white dwarfs (the most common) the few available $\mathrm{H}$ and $\mathrm{He}$ lines do not allow one to reach the required precision of $<1 \mathrm{~m} \mathrm{~s}^{-1}$.

However, a planet in the CWD habitable zone would reflect a relatively large fraction of the stellar flux, producing also a relatively strong polarisation signal. Therefore, reflected light (with ellipsoidal variation) and polarisation (Seager et al. 2000) seem to be the most viable ways to detect close-in non-transiting rocky planets orbiting white dwarfs.

As shown by Seager et al. (2000) and Stam (2008), as a planet orbits around the host star, the amount of polarisation varies regularly, showing maxima when the planet is near quadrature. The amplitude of the variation depends mainly on the orbital inclination, $i$, with no polarimetric diurnal variability for a face-on orbit. The detection and measurement of regular polarisation variations allows the discovery of exoplanets, with an efficiency dependent on $i$, similar to that of the radial velocity planet detection methods. Spectropolarimetry of planet-hosting stars could also allow one to characterise the atmosphere of an exoplanet (see e.g. Stam 2008; Sterzik et al. 2012).

As a matter of fact, polarimetric measurements have already been obtained for a few hot-Jupiters, but with contradicting results. Berdyugina et al. (2011) reported the first detection of polarised reflected light from HD 189733b with the DiPol and TurPol instruments of the Nordic Optical Telescope (NOT) at a level of $10^{-4} \pm 10^{-5}$ in the $U$ and $B$ photometric bandpasses. They used their measurements to constrain various planetary and orbital parameters, such as planetary albedo and orbital inclination. This result has been challenged, as such a high polarisation implies an unusually big radius and albedo of this hot-Jupiter. The detection was not corroborated by precision polarimetry with the POLISH instrument mounted at the Cassegrain of the 5-m Hale/Palomar telescope in a broad, visual bandpass region, as Wiktorowicz (2009) report an upper limit on the polarisation level of $<7.9 \times 10^{-5}$. Similarly, Lucas et al. (2012) failed to detect polarimetric variations in two other promising systems hosting hot-Jupiters, 55 Cnc and 
$\tau$ Boo, reaching accuracy limits of $\leqslant 5 \times 10^{-6}$. Kostogryz et al. (2011) attribute the detection of Berdyugina et al. (2011) to (stellar) polarisation caused at the limb during planetary transit, but the correct interpretation remains unclear.

Using white dwarf model fluxes, computed with the stellar atmosphere code developed by Kowalski (2006) and Kowalski \& Saumon (2006), and models of reflected light and degree of polarisation from Stam (2008), Fossati et al. (2012) estimated the amount of polarised light reflected by the atmosphere of an unresolved Earth-sized planet in the CHZ of CWDs. For all calculations they assumed a $\mathrm{CO}$ white dwarf of radius $0.013 R_{\odot}$ and mass of $0.6 M_{\odot}$, the angle between star and Earth as seen from the planet $(\alpha)$ equal to 0.68 (where the degree of polarisation is expected to be maximum), an orbit inclination angle of $90^{\circ}$, and a cloud-free Lambertian planet surface with a wavelength independent albedo of 1.0 (labelled as "L10" by Stam 2008). Following Stam (2008), Fossati et al. (2012) concluded that the maximum polarisation $\left(P_{\mathrm{obs}, \max }(\lambda)\right)$ can be defined as:

$$
P_{\mathrm{obs}, \max }(\lambda) \approx \frac{r^{2}}{d^{2}} \frac{1}{4} b_{1}\left(\lambda, \alpha=68^{\circ}\right)
$$

where $r$ and $d$ are, respectively, the planet radius and orbital separation, and $b_{1}(\lambda, \alpha)$ is the element of the planet scattering matrix providing the information on the polarisation (e.g. Stam et al. 2006).

In their Fig. 3, Fossati et al. (2012) show the maximum polarisation, as a function of wavelength, produced by an unresolved Earth-sized planet orbiting at a distance of $0.01 \mathrm{AU}$ from a $5000 \mathrm{~K}$ white dwarf, and examined also the dependence of the polarisation on orbital separation and planet radius (at a reference wavelength of $4000 \AA$ ). They additionally compared the level of polarisation emitted by the same planet placed in the habitable zone of a Sun-like star and of a typical M dwarf, concluding that the polarisation signal of any planet in the white dwarf $\mathrm{CHZ}$ is larger than that of a comparable planet in the habitable zone of any other type of star. The polarisation produced by an unresolved Earth-sized planet at $1 \mathrm{AU}$ from a Sun-like star is the order of $10^{-10}$ and for a typical M dwarf, for which the habitable zone is at a distance of $\sim 0.1 \mathrm{AU}$ (Selsis et al. 2007), the amount of polarisation is of the order of $10^{-8}$, while in the case of a CWD the amount of polarisation is the order of $10^{-6}$.

\section{Detectability of polarised light from Earth-like planets in the white dwarf CHZ}

The signal-to-noise ratio $(\mathrm{S} / \mathrm{N})$ of the observations that is required to detect the polarisation signal (at $3 \sigma$ ) of a rocky planet in the white dwarf CHZ is of $\sim 10^{6}$. The largest obstacle in reaching such a high $\mathrm{S} / \mathrm{N}$ is the fact that white dwarfs are generally faint, mostly because of their small radius. In addition, the very short planet orbital period ( 12 hours in the CHZ; Agol 2011) limits integration times to 2-3 hours.

Figure 2 shows the polarisation detectable within an exposure time of 2.5 hours with a hypothetical polarimeter (having characteristics similar to those of the FORS2 instrument at the ESO/VLT) mounted on a 8-m, 40-m, and 100-m telescope as a function of wavelength (i.e. in the $B, V$, and $R$ bands) and stellar magnitude. As already shown by Berdyugina et al. (2011), the $B$ band polarisation compares well with that obtained in the $V$ and $R$ bands, because of the larger polarisation emitted by the planet at shorter wavelengths, despite the lower flux and CCD sensitivity. On the other hand, the severe drop in the CCD sensitivity makes the reachable $\mathrm{S} / \mathrm{N}$ in the $U$ band far too low.

The brightest single CWD is WD $0046+051\left(T_{\text {eff }}=6220 \mathrm{~K}\right)$, with a magnitude of $V \sim$ 12.4 (Holberg et al. 2008). The available observing facilities are capable of a $3 \sigma$ detection 


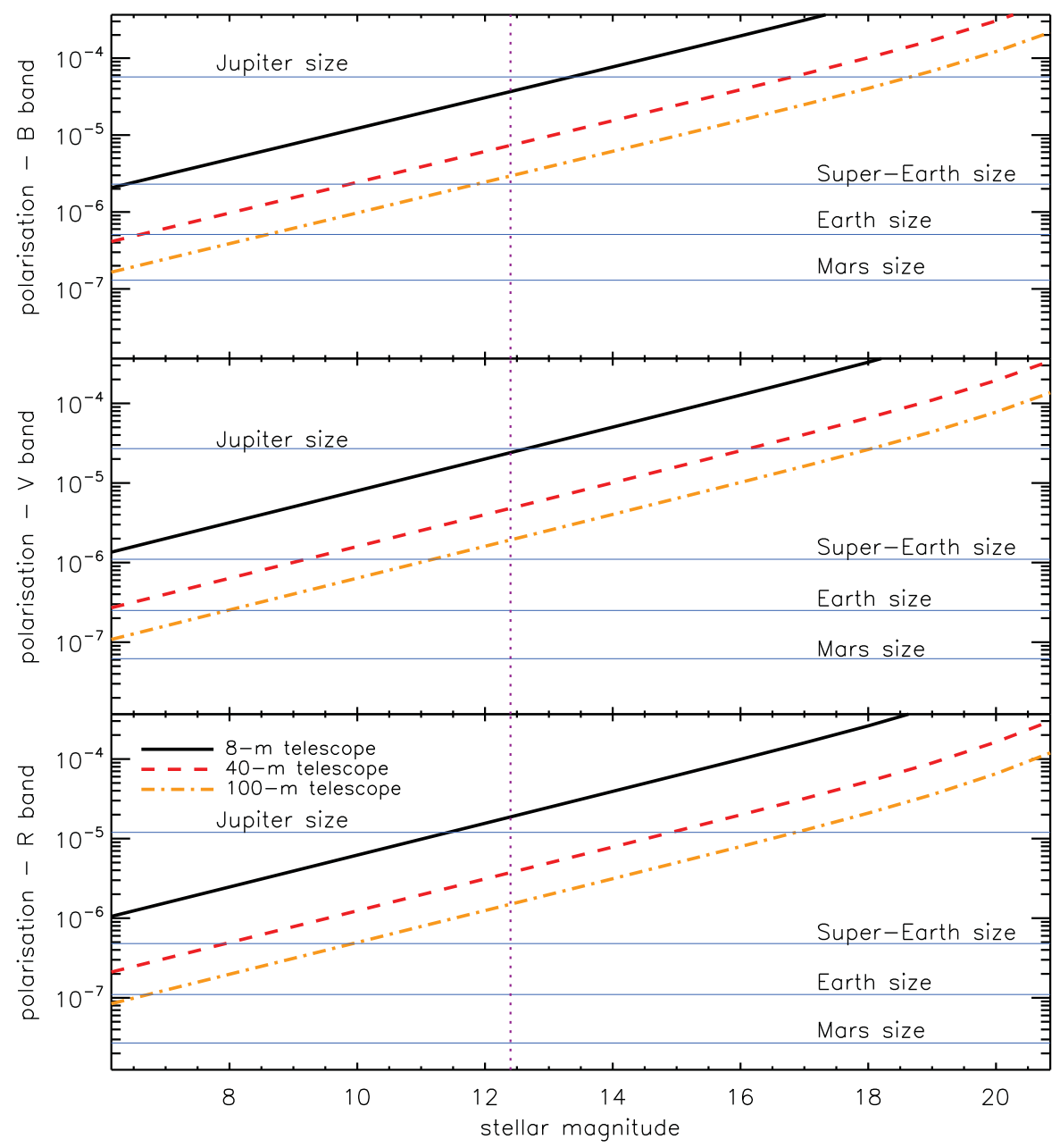

Figure 2. Amount of polarisation detectable at $3 \sigma$ in the $B$ (top), $V$ (middle) and $R$ (bottom panel) bands, as a function of stellar magnitude, with a hypothetical FORS-like polarimeter mounted on a 8-m (black full line), 40-m (red dashed line), and 100-m (yellow dash-dotted line) telescope, within an exposure time of 2.5 hours. The horizontal lines show the amount of polarisation emitted in the three bands by planets of different sizes, orbiting at $0.01 \mathrm{AU}$ from a $T_{\text {eff }}=5000 \mathrm{~K}$ CWD. The dotted purple vertical line indicates the magnitude of WD $0046+051$, the brightest known cool white dwarf. Figure adapted from Fossati et al. (2012).

only of a Jupiter-sized planet in the CHZ of this white dwarf, while with future observing facilities we would be able to detect super-Earth-sized planets (between 2.0 and $2.5 R_{\oplus}$ ), in the CHZ. All other known white dwarfs, with an effective temperature below $6000 \mathrm{~K}$, are all fainter than $V \sim 14 \mathrm{mag}$ and Fig. 2 shows that even a $40-\mathrm{m}$ telescope (e.g. E-ELT) would be able to barely detect super-Earth-sized planets in the CHZ, via polarimetry.

The detection threshold of the polarisation emitted by a planet in the habitable zone of CWDs could be improved for example by using filters with a very broad wavelength coverage. In this way, one would lose the spectral information, but gain considerably in number of collected photons. It would be also very important to use instruments with an improved sensitivity in the blue, compared to what is currently available. As an example, the FORS2 instrument in the currently available standard setup is used with a CCD that has its sensitivity peak in the red. Note that this is also the CCD that has 
been considered for the detectability presented by Fossati et al. (2012) and shown in Fig. 2. In this way, it would be possible to detect (at $3 \sigma$ ) a super-Earth-sized planet in the CHZ of WD 0046+051, the brightest single white dwarf, with the currently available facilities.

It is also important to notice that the level of polarisation calculated by Fossati et al. (2012) and further considered here was that of a purely spherical planet, while Fossati et al. (2012) showed that planets in the CHZ of CWDs would fill a substantial fraction of their Roche lobes, making the planet atmosphere more extended and with a teardropshape. In this way, the area of the planet actually reflecting the stellar light close to quadrature should be larger compared to what considered in Fig. 2.

The search for directly planet-reflected light would also increase the chances to detect planets in the habitable zone of CWDs. As shown by Seager et al. (2000) at phase angle $\alpha=0$, (orbital phase 0.5 , superior conjunction of the planet) the full disc of a planet at $i=90^{\circ}$ will be illuminated producing a photometric maximum. The photometric minimum occurs at inferior conjunction of the planet $\left(\alpha=180^{\circ}\right)$, with intermediate fluxes at quadrature $\left(\alpha=90^{\circ}\right)$, where instead the polarimetric maxima occur. The detailed photometric light curve would depend on the planet scattering matrix, but the reflected flux will be roughly proportional to the illuminated area. Photometric variability can, however, arise from many different mechanisms, so a planet detection based on photometry alone would not be credible. Since cool non-magnetic white dwarfs are expected to have little intrinsic photometric variability, a small photometric signal phased appropriately with the polarimetric variability would provide the necessary confirmation to a polarimetric planet detection.

\section{Acknowledgements}

LF acknowledges financial support from the Alexander von Humboldt foundation. GV acknowleges the Russian Scientific Foundation (grant N14-50-00043)

\section{References}

Agol, E. 2011, ApJ (Letters) 731, L31

Barnes, R. \& Heller, R. 2013, Astrobiology 13, 279

Bear, E. \& Soker, N. 2013, New Astronomy 19, 56

Berdyugina, S. V., Berdyugin, A. V., Fluri, D. M., \& Piirola, V. 2011, ApJ (Letters) 728, L6

Bergfors, C., Farihi, J., Dufour, P., \& Rocchetto, M. 2014, MNRAS 444, 2147

Bonsor, A., Mustill, A. J., \& Wyatt, M. C. 2011, MNRAS 414, 930

Charpinet, S., Fontaine, G., Brassard, P., Green, E. M., Van Grootel, V., Randall, S. K., Silvotti, R., Baran, A. S., Østensen, R. H., Kawaler, S. D., \& Telting, J. H. 2011, Nature 480, 496

Debes, J. H., Walsh, K. J., \& Stark, C. 2012, ApJ 747, 148

Eggleton, P. P. 1971, MNRAS 151, 351

Faedi, F., West, R. G., Burleigh, M. R., Goad, M. R., \& Hebb, L. 2011, MNRAS 410, 899

Farihi, J., Jura, M., \& Zuckerman, B. 2009, ApJ 694, 805

Farihi, J., Gänsicke, B. T., Steele, P. R., Girven, J., Burleigh, M. R., Breedt, E., \& Koester, D. 2012, MNRAS 421, 1635

Fossati, L., Bagnulo, S., Haswell, C. A., Patel, M. R., Busuttil, R., Kowalski, P. M., Shulyak, D. V., \& Sterzik, M. F. 2012, ApJ (Letters) 757, L15

Frewen, S. F. N. \& Hansen, B. M. S. 2014, MNRAS 439, 2442

Friedrich, S., Zinnecker, H., Correia, S., Brandner, W., Burleigh, M. \& McCaughrean, M. 2007, in: R. Napiwotzki \& R. Burleigh (eds.), Search for Giant Planets around White Dwarfs with HST, Spitzer, and VLT, Proc. 15th European Workshop on White Dwarfs (ASP-CS), p. 372 
Frink, S., Mitchell, D. S., Quirrenbach, A., Fischer, D. A., Marcy, G. W., \& Butler, R. P. 2002, ApJ 576, 478

Fulton, B. J., Tonry, J. L., Flewelling, H., Burgett, W. S., Chambers, K. C., Hodapp, K. W., Huber, M. E., Kaiser, N., Wainscoat, R. J., \& Waters, C. 2014, ApJ 796, 114

Gänsicke, B. T., Marsh, T. R., Southworth, J., \& Rebassa-Mansergas, A. 2006, Science 314, 1908

Haswell, C. 2010, 'Transiting exoplanets', CUP ISBN 978-0521139380

Hatzes, A. P., Guenther, E. W., Endl, M., Cochran, W. D., Döllinger, M. P., \& Bedalov, A. 2005, A\&SA 437, 743

Holberg, J. B., Sion, E. M., Oswalt, T., McCook, G. P., Foran, S., \& Subasavage, J. P. 2008, AJ 135,1225

Koester, D., Gänsicke, B. T., \& Farihi, J. 2014, A\& A 566, A34

Kostogryz, N. M., Yakobchuk, T. M., Morozhenko, O. V., \& Vid'Machenko, A. P. 2011, MNRAS 415,695

Kowalski, P. M. 2006, PhD Thesis, Vanderbilt University, whitedwarf.org/theses/ kowalski.pdf

Kowalski, P. M. \& Saumon, D. 2006, ApJ 651, L137

Lai, D. 2012, ApJ (Letters) 757, L3

Li, J., Ferrario, L., \& Wickramasinghe, D. 1998, ApJ (Letters) 503, L151

Lucas, P. W., Hough, J. H., Bailey, J. A., Tamura, M., Hirst, E., \& Harrison, D. 2009, MNRAS 393,229

McCree, K. J. 1972, Agric. Meteorol. 10, 443

Melis, C., Dufour, P., Farihi, J., Bochanski, J., Burgasser, A. J., Parsons, S. G., Gänsicke, B. T., Koester, D., \& Swift, B. J. 2012, ApJ (Letters) 751, L4

Monteiro, H. 2010, Bulletin of the Astronomical Society of Brazil 29, 22

Mullally, F., Winget, D. E., De Gennaro, S., Jeffery, E., Thompson, S. E., Chandler, D., \& Kepler, S. O. 2008, ApJ 676, 573

Patel, M. R., Christou, A. A., Cockell, C. S.,Ringrose, T. J., \& Zarnecki, J. C. 2004, Icarus 168, 93

Raven, J. 2007, Nature 488, 418

Salaris, M., Cassisi, S., Pietrinferni, A., Kowalski, P. M., \& Isern, J. 2010, ApJ 716, 1241

Sato, B., Ando, H., Kambe, E., Takeda, Y., Izumiura, H., Masuda, S., Watanabe, E., Noguchi, K., Wada, S., Okada, N., Koyano, H., Maehara, H., Norimoto, Y., Okada, T., Shimizu, Y., Uraguchi, F., Yanagisawa, K., \& Yoshida, M. 2003, ApJ (Letters) 597, L157

Seager, S., Whitney, B. A., \& Sasselov, D. D. 2000, ApJ 540, 504

Selsis, F., Kasting, J. F., Levrard, B., Paillet, J., Ribas, I., \& Delfosse, X 2007, A $\& A$ 476, 1373

Silvotti, R., Schuh, S., Janulis, R., Solheim, J.-E., Bernabei, S., Østensen, R., Oswalt, T. D., Bruni, I., Gualandi, R., Bonanno, A., Vauclair, G., Reed, M., Chen, C.-W., Leibowitz, E., Paparo, M., Baran, A., Charpinet, S., Dolez, N., Kawaler, S., Kurtz, D., Moskalik, P., Riddle, R., \& Zola, S. 2007, Nature 449, 189

Silvotti, R., Charpinet, S., Green, E., Fontaine, G., Telting, J. H., Østensen, R. H., Van Grootel, V., Baran, A. S., Schuh, S., \& Fox Machado, L. 2014, A\&SA 570, A130

Stam, D. M., de Rooij, W. A., Cornet, G., \& Hovenier, J. W. 2006, A 6 A 452, 669

Stam, D. M. 2008, A\&BA 482, 989

Stancliffe, R. J., Glebbeek, E., Izzard, R. G., \& Pols, O. R. 2007, A $\mathscr{E} A$ 464, L57

Sterzik, M. F., Bagnulo, S., \& Palle, E. 2012, Nature 483, 64

Valyavin, G., Shulyak, D., Wade, G. A., Antonyuk, K., Zharikov, S. V., Galazutdinov, G. A., Plachinda, S., Bagnulo, S., Fox Machado, L., Alvarez, M., Clark, D. M., Lopez, J. M., Hiriart, D., Han, I., Jeon, Y.-B., Zurita, C., Mujica, R., Burlakova, T., Szeifert, T., \& Burenkov, A. 2014, Nature 525, 88

Veras, D., Leinhardt, Z. M., Bonsor, A., \& Gänsicke, B. T. 2014, MNRAS 445, 2244

Veras, D. \& Gänsicke, B. T. 2014, MNRAS 447, 1049

Wiktorowicz, S. J. 2009, ApJ 696, 1116

Zuckerman, B., Melis, C., Klein, B., Koester, D., \& Jura, M. 2010, ApJ 722, 725 